\title{
Medienpädagogik in der katholischen Erwachsenenbildung
}

von Georg Betz

Seit Jahren wird nach ihr gerufen. Kaum ein Aufsatz, keine Tagung über Probleme, Chancen und Risiken der Massenmedien, an deren Ende nicht der Verweis auf die Dringlichkeit von „Medienerziehung” steht, mögen die Begründung, Zielvorstellungen, Inhalte und die Begrifflichkeit auch beträchtlich divergieren. Der Ruf ist bekanntlich nicht ungehört verhallt: Lehrstühle haben sich etabliert. In den Lehrplänen für das allgemeinbildende Schulwesen sind die Medien zu Unterrichtsgegenständen erhoben. Die Medien selbst beteiligen sich am Versuch ihrer Bewältigung. Und schließlich sehen sich auch die Einrichtungen der Erwachsenenbildung in Sachen Medienerziehung besonders gefordert.

\section{Medienerziehung im Selbstverständnis katholischer Erwachsenenbildung}

Im Selbstverständnis der Erwachsenenbildung in katholischer Trägerschaft kommt der Medienerziehung ein hoher Stellenwert zu. Franz Henrich, Direktor der Katholischen Akademie in Bayern und mittlerweile Vorsitzender der Katholischen Bundesarbeitsgemeinschaft für Erwachsenenbildung, sieht in der Medienerziehung ausdrücklich einen jener „sieben großen Themenbereiche”, die „sich vor allem für die Erwachsenenbildung in katholischer Trägerschaft anbieten"'. Henrich geht dabei davon aus, daß die plurale Verfassung der Erwachsenenbildung in der Bundesrepublik „Arbeitsteilung” erfordere, „Konzentration eines Trägers auf bestimmte Sachbereiche".

In Henrichs Vorstellungen von einem ,schwerpunktmäßig reduzierten Angebot” wird neben dem „Bereich des Politischen, Gesellschaftlichen, Wirtschaftlichen, Sozialen”, neben dem „pädagogisch-psychologischen Bereich”, dem „naturwissenschaftlich-technischen Bereich", dem philosophischen sowie dem religiös-theologisch-kirchlichen Bereich”, dem „kulturell-musischen Bereich” und neben dem „berufsorientierten Bereich” ausdrücklich „der Bereich der Medienerziehung” aufgeführt. Henrich deutet wie bei den anderen Bereichen dabei auch „stichwortartig”, "zeitbedingt" und ohne Anspruch auf Systematik und Vollständigkeit den „Akzent” an,,unter dem die Themen in unseren Tagen gerade von Bildungswerken, die von Christen getragen sind, behandelt werden sollten als ihr spezifischer Beitrag im Gesamtangebot der Erwachsenenbildung”. Es heißt dort für den medienpädagogischen Bereich: „Themenkomplexe: Buch, Presse, Rundfunk, Fernsehen, Kassette, Medienkunde usw. - Hinführung zu vernünftigem Gebrauch, zu Kritik, Urteil und eigenem Denken. Akzente: Information oder Manipulation?, Unterhaltung oder Verblödung?; ästhetische und ethische Kategorien,”

Beim grundsätzlichen Recht auf inhaltliche Universalität sieht der „Kirchliche Erwachsenenbildungsplan der Erzdiözese München” ebenfalls „Medienerziehung” ausdrücklich als eins von acht „Stoffgebieten” an, ,in denen die Einrichtungen der Erwachsenenbildung in katholischer Trägerschaft Angebote bereitstellen"2. Als inhaltliche „Schwerpunkte” sind dort benannt: „Film, Funk, Fernsehen, Presse”.

Dr. Georg Betz ist wissenschaftlicher Assistent am Lehrstuhl Erwachsenenbildung der Katholischen Universität Eichstätt. 
Als „Ziel” wird angegeben: „Hinführung zum vernünftigen Gebrauch der Medien, Zu Kritik, Urteil und Distanz."

Im „Plan für die offene Erwachsenenbildung in der Diözese Eichstätt” wird als ein „Schwerpunkt katholischer Erwachsenenbildung” ebenfalls eigens ein „Bereich Massenmedien” benannt: „Katholische Erwachsenenbildung”, so die Erläuterung der Zielperspektive, ,will helfen, daß der einzelne Maßstäbe zur vernünftigen Auswahl und zum kritischen Gebrauch der vielfältigen Angebote von Film, Hörfunk, Fernsehen, Presse, Schallplatte, Buch gewinnt und seine Rolle im Zeitgespräch der Gesellschaft erkennt und aktiv wahrnimmt."

Wo derlei ausdrückliche Verweise auf Medienpädagogik als eine Schwerpunktaufgabe katholischer Erwachsenenbildung fehlen, ist sie zumindest implizit angelegt in der (den) Zielperspektive(n), die katholischer Erwachsenenbildung zugeschrieben werden: Wenn sie den Menschen ,in einer Weise ausrüsten will, daß er 'nicht manipuliertes Objekt, sondern mitgestaltendes Subjekt gesellschaftlichen Lebens zu sein“ vermag”4, wenn sie „Orientierungsmöglichkeiten” vermitteln soll, „die zur kritischen Haltung befähigen und so die mündige und informierte Teilnahme am gesellschaftlichen und politischen Leben fördern"s, dann stellt die Hilfestellung zum sachgerechten, selbstverantwortlichen, zum „mündigen” Umgang mit den Medien sozialer Kommunikation eine der wichtigen Konkretionen solcher allgemeinen Zielperspektive(n) dar.

\section{Ein kirchlicher Auftrag}

Im übrigen entspricht die katholische Erwachsenenbildung offiziellen kirchenamtlichen Forderungen, wenn sie Medienerziehung zu einer ihrer zentralen Aufgaben erklärt. In der „Pastoralinstruktion Communio et progressio über die Instrumente der soziaen Kommunikation” sieht die Kirche die „dringliche Notwendigkeit”, sich selbst in der Medienerziehung zu engagieren. Den „katholischen Schulen und Bildungseinrichtungen" wird darin ein „schwerwiegender Auftrag auf diesem Gebiet” zugesprochen ${ }^{6}$. Das Pastoralschreiben hat zugleich die Einlösung dieser Verpflichtung seitens der katholischen Einrichtungen nicht deren Beliebigkeit anheimgestellt. Es hat Grundsätze einer soliden Medienerziehung entwickelt, der Bildungspraxis also Qualitätsmaßstäbe vorgegeben. Formal wird von einer guten Medienerziehung erwartet, daß sie ,jeweils den neuesten Stand der Entwicklung berücksichtigt, dem Alter der Rezipienten angepaßt und ausreichend ist". Ein solches Angebot sollte für alle „leicht zugänglich" und es sollte „fortschreitend”, gestuft in Grund- und Aufbaukurse, angelegt sein?.

Von der Zielsetzung her, inhaltlich, vollzieht das Pastoralschreiben eine Abkehr von einer Medienerziehung, die nur bewahrenden Charakter hat, indem sie vor den Risiken der Mediennutzung warnt. Die im Pastoralschreiben skizzierte "rechte” Medienerziehung will demgegenüber vorrangig mithelfen, „da $ß$ jeder seinen Beitrag zum Gespräch und zur schöpferischen Zusammenarbeit in der Gesellschaft leisten kann"8, daß also jeder fähig wird, seine Rolle als Kommunikationspartner im Gespräch der Gesellschaft zu sehen und aktiv wahrzunehmen.

Hans Wagner hat in seinem Kommentar zum Pastoralschreiben die inhaltlichen Konsequenzen einer so verstandenen Medienpädagogik skizziert: Sie müsse gründlich vermitteln, was an Grundsätzlichem in der Instruktion über Wesen und Funktionsweisen der Instrumente der sozialen Kommunikation ausgesagt ist. Sie müsse 
des weiteren die Eigenart der einzelnen Medien, ihre Organisationsform und Struktur bewußt machen. Und nicht zuletzt habe sie die Grundsätze für den sachgerechten Umgang mit Kommunikationsmedien aufzuzeigen, immer im Blick auf den einzelnen wie die ganze Gesellschaft ${ }^{9}$.

\section{Scblaglichter auf die Wirklichkeit}

Medienpädagogik ist Programm und Verpflichtung katholischer Erwachsenenbildung. Ist sie aber auch ihre Realität? Wird praktiziert, was katholische Erwachsenenbildung zu einer ihrer zentralen Aufgaben erklärt bzw. was ihr kirchlicherseits als solcher aufgetragen wurde? Und wie wird Medienpädagogik in der katholischen Erwachsenenbildung betrieben? Entsprechend den im Pastoralschreiben entwickelten Qualitätsmaßstäben? Folgt man Franz Henrich, so ist es um die Medienerziehung der katholischen Erwachsenenbildung nicht gut bestellt: „In der Medienerziehung”, so Henrich, ,sind wir mit bedeutenderen Programmen bislang noch nicht hervorgetreten”. Wir reden zwar ab und zu über die „Gewalt im Fernsehen”, gelangten aber noch nicht zu einer systematischen Schulung unserer Zuhörer." 10 Trifft Henrichs pauschale Charakterisierung die Wirklichkeit? Was läuft an medienpädagogischen Aktivitäten in der katholischen Erwachsenenbildung? Welchen Umfang hat das Angebot? Wie sieht es formal und inhaltlich aus?

Im folgenden wird darauf eine erste noch ergänzungs- und differenzierungsbedürftige Antwort versucht. Ergänzungs- und differenzierungsbedürftig ist die Antwort deshalb, weil sie nur die Aktivitäten katholischer Erwachsenenbildung vor Ort, also die Bildungsarbeit in den Gemeinden, Städten oder auf Kreisebene im Auge hat, die typische Abend-Erwachsenenbildung. Sich auf diesen Sektor katholischer Erwachsenenbildung zunächst zu konzentrieren, erschien besonders relevant: Katholische Bildungsarbeit hat vor Ort einen hohen Grad von Flächendeckung erreicht und operiert deshalb relativ bürgernah, also auch besonders nah an jenen Bevölkerungsschichten, die den Weg in ein Bildungshaus oder eine Akademie nicht finden, bei denen aufgrund ihrer Bildungsgeschichte und Lebenssituation im allgemeinen aber besonders große Bewußtseinsdefizite in Sachen Medien vermutet werden dürfen.

Ergänzungs- und differenzierungsbedürftig ist die Antwort auch deshalb, weil sie nicht auf einer Totalerhebung aller Aktivitäten der örtlichen Einrichtungen basiert und vor allen Dingen nicht das Geschehen während der Veranstaltungen erfaßt. Antwortbasis sind zum einen inhaltlich grob aufgeschlüsselte Statistiken über die von Einrichtungen der Katholischen Erwachsenenbildung durchgeführten Bildungsmaßnahmen sowie eine eigene Analyse von Programmheften katholischer Kreis- bzw. Stadtbildungswerke aus verschiedenen Bundesländern, vorwiegend aber aus dem süddeutschen Raum. Vorhandensein bzw. Zugänglichkeit haben die Auswahl der Programmhefte weit mehr geleitet als der Wunsch nach Herstellung bundesweiter Repräsentativität.

Der Analyse wurde das Veranstaltungsverzeichnis über das erste Programmhalbjahr 1980 (I/80) von insgesamt 40 Bildungswerken zugrunde gelegt, dazu von 20 dieser 40 Bildungswerke auch die beiden Veranstaltungsverzeichnisse des Jahres I979 (II/79) und (I/79). Schließlich wurde noch das örtliche Programmangebot in hundert nach dem Zufallsprinzip ausgewählten Nebenstellen bzw. Gemeinden/ Pfarreien über die letzten fünf Programmjahre (von II/75 bis I/80) hinweg 
gesondert gesichtet. Allerdings rekrutierten sich diese hundert Gemeinden aufgrund des für eine Längsschnittanalyse verfügbaren Materials lediglich aus zwölf Stadt- bzw. Kreisbildungswerken, und nur noch aus dem bayerischen Raum. Doch dürfte auch eine solch schmale Materialbasis - die weiteren Ausführungen werden das zeigen - ein bemerkenswertes Schlaglicht auf die Realität der Medienerziehung im Gesamt der Aktivitäten katholischer Erwachsenenbildung werfen.

\section{Zum Umfang des medienpädagogischen Angebots}

$\mathrm{Daß}$ medienpädagogische Aktivitäten innerhalb des Veranstaltungsangebots katholischer Erwachsenenbildung vor Ort nur einen sehr bescheidenen Raum einnehmen, lassen bereits die offiziellen Leistungsstatistiken katholischer Erwachsenenbildung, soweit sie eine Kategorie Medien führen, ablesen. Nach der amtlichen bayerischen Landesstatistik ${ }^{11}$ sind 1978 in ganz Bayern 306 Veranstaltungen zum Bereich „Medien, Film, Funk, Fernsehen, Presse” durchgeführt worden. Bezogen auf das Gesamt aller für die katholische Erwachsenenbildung in Bayern ausgewiesenen Maßnahmen waren das 0,86\%. Die 651 Doppelstunden (1 Doppelstunde entspricht 90 Minuten), die dabei an Auseinandersetzung mit der Medienproblematik zusammen kamen, machten gerade $0,6 \%$ am Gesamt der seitens katholischer Bildungseinrichtungen in Bayern durchgeführten Doppelstunden aus.

Ein Jahr zuvor waren es 401 Veranstaltungen $(=1,29 \%)$ und 734 Doppelstunden $(=0,72 \%)$. Innerhalb eines Jahres hat sich demnach eine deutliche Schrumpfung des medienpädagogischen Angebots im Programm der katholischen Erwachsenenbildung in Bayern vollzogen, und das bei gleichzeitiger starker Expansion der Veranstaltungen und Doppelstundenzahlen. 1977 steuerten die kath. Weiterbildungseinrichtungen mit den oben genannten Werten zur medienpädagogischen Gesamtleistung der öffentlichen bayerischen Erwachsenenbildung 26,7\% der Veranstaltungen und 19,8\% der Doppelstunden bei, Anteile, die sich im Rahmen der Größenordnung des gesamten kath. Bildungsbeitrags in diesem Jahr bewegten ${ }^{12}$. Mit ihrem medienpädagogischen Engagement tut sich katholische Erwachsenenbildung quantitativ unter den anerkannten öffentlichen Weiterbildungsträgern nicht hervor.

Auch die sehr differenzierte hessische Landesstatistik ${ }^{13}$ läßt Rückschlüsse auf den Umfang des medienpädagogischen Beitrags seitens der katholischen Erwachsenenbildung zu. Unter der Kategorie „Medien (Film, Fernsehen)” werden dort für das Jahr 1978 insgesamt 47 Veranstaltungen mit zusammen 323 Unterrichtsstunden (à 45 Minuten) ausgewiesen. In Anteilen am Gesamtprogramm umgerechnet waren das $0,62 \%$ aller Veranstaltungen und $0,28 \%$ aller Unterrichtsstunden. Selbst wenn man in Hessen auch die Kategorie „Medientechnik”, in der Anleitungen zum Fotografieren, Filmen oder zum Umgang mit dem Tonband gegeben werden, als medienpädagogische Aktivität werten wollte, bleibt, da $B$ Medienerziehung auch in Hessen nur ein bescheidenes Randdasein fristet.

Für einen Teil Hessens, das Bistum Limburg, liegen auch Zahlen aus vorhergehenden Jahren vor ${ }^{14}$. Danach dürfte der bescheidene Stellenwert der Medienpädagogik im faktischen Programmangebot nicht auf eine 1978 in Hessen eingelegte besondere medienpädagogische Pause zurückgehen. Er ist eher Niederschlag einer medienpädagogischen Dauerabstinenz. Von der Gesamtzahl an Unterrichtseinheiten entfielen $19750,78 \%, 19760,83 \%, 19770,50 \%$ und $19780,53 \%$ auf das Stoffgebiet „Medienkunde (Film, Funk, Fernsehen usw.)". 
Ganz Ähnliches zeigt die Leistungsstatistik der katholischen Erwachsenenbildung in der Erzdiözese Freiburg ${ }^{15} .1972$ entfielen danach dort 142 Unterrichtseinheiten à 45 Minuten auf medienpädagogische Veranstaltungen. Das waren $0,92 \%$ aller durchgeführten Unterrichtseinheiten. 1973 waren es 318 Unterrichtseinheiten $(=1,72 \%), 1974389(=1,74 \%), 1975196(=0,73 \%), 1976150(=0,45 \%), 1977334$ $(=0,67 \%)$ und $1978220(=0,38 \%)$. Während sich die gesamten Bildungsaktivitäten in diesem Zeitraum in der Erzdiözese Freiburg nahezu vervierfacht haben, hat sich der Umfang des medienpädagogischen Angebots aufs Ganze gesehen nicht ausgeweitet. Das medienpädagogische Engagement hat mit der Expansion katholischer Erwachsenenbildung nicht Schritt gehalten. Medienerziehung hat faktisch im Programm katholischer Erwachsenenbildung an Terrain verloren.

Bei aller notwendigen Problematisierung der Analyse von Grobstatistiken bleibt dabei immerhin auch zu bedenken, da $\beta$ in das von ihnen ausgewiesene verschwindend geringe medienpädagogische Angebot teilweise auch die Aktivitäten der Bildungshäuser im Einzugsbereich eingehen und deshalb die Veranstaltungen und Unterrichts- bzw. Doppelstundenzahlen vor Ort eher noch etwas niedriger angesetzt werden müssen, daß also die Leistungsstatistiken die Kargheit des medienpädagogischen Angebots katholischer Erwachsenenbildung vor Ort eher noch etwas kaschieren.

Die Analyse der Veranstaltungsverzeichnisse von 40 regionalen Bildungswerken über das Arbeitshalbjahr I/80 bestätigt die schon aus der Grobstatistik abgeleitete Geringfügigkeit des medienpädagogischen Engagements im Rahmen der breitangelegten Bildungsbemühungen katholischer Erwachsenenbildung vor Ort. Unter den dabei gesichteten 10.108 geplanten Bildungsmaßnahmen konnten ganze 63 registriert werden, bei denen es um Massenmedien gehen sollte. Das sind $0,62 \%$. In denselben Veranstaltungsverzeichnissen wurden zugleich 42 öffentlich angekündigte Veranstaltungseinheiten registriert, in deren Mittelpunkt „Heilkräuter” stehen sollten. In 11 der 40 Bildungswerke waren im 1. Halbjahr 1980 überhaupt keine medienpädagogischen Veranstaltungen angesetzt, in 11 nur jeweils eine, und nur in neun Bildungswerken drei und mehr.

\section{Zur Intensität medienpädagogischer Angebote}

Bezüglich der zeitlichen Intensität der Beschäftigung mit medienpädagogisch relevanten Sachverhalten erlauben bereits Grobstatistiken gewisse Rückschlüsse:

Das deutliche Zurückfallen des Doppel-bzw. Unterrichtsstundenanteils hinter den Anteil von abgeschlossenen Maßnahmen in Bayern wie in Hessen kann bereits andeuten, daß es sich zumeist nicht um besonders lernintensive, langfristig angelegte Veranstaltungen handelt. In Bayern errechnet sich für die medienpädagogische Durchschnittsveranstaltung im Jahre 1978 eine Länge von 2,11 Doppelstunden pro Veranstaltung. Zum Vergleich: Bezogen auf das Gesamt aller Bildungsmaßnahmen der katholischen Erwachsenenbildung in Bayern belief sich die durchschnittliche Veranstaltungsdauer im selben Jahr auf 3,42 Doppelstunden. In Hessen hatte das medienpädagogische Durchschnittsangebot eine Dauer von 3,44 Doppelstunden und lag damit beträchtlich hinter der Gesamtdauer von 7,45 Doppelstunden im Schnitt aller durchgeführten Maßnahmen zurück ${ }^{16}$.

Sowohl in Hessen wie in Bayern bestand 1978 das medienpädagogische Angebot großenteils aus „Einzelveranstaltungen”. In Hessen waren von den 47 unter der 
Kategorie „Medien” geführten Angeboten nur 9 längerfristige Unternehmen: 4 Kurse mit zusammen 23 Doppelstunden und 5 Kompaktkurse, die über mindestens zwei Veranstaltungstage mit jeweils mindestens acht Doppelstunden bei mindestens einer Übernachtung liefen. Rund $80 \%$ aller medienpädagogischen Angebote im Programm der hessischen katholischen Erwachsenenbildung waren also Einzelveranstaltungen. In Bayern entfielen 1978 von den 308 thematisch abgeschlossenen medienpädagogischen Maßnahmen $61 \%$ auf „Abend-Einzelveranstaltungen” und knapp $15 \%$ auf „Halbtagsveranstaltungen”. Über mehr als zwei Doppelstunden hinweg befaßten sich mit Fragestellungen aus dem Medienbereich also nicht einmal ganz ein Viertel der medienpädagogischen Angebote.

Auch bezüglich der zeitlichen Intensität schönt die amtliche Leistungsstatistik mit der teilweisen Berücksichtigung der zumeist zeitlich längerfristig angelegten Angebote der Bildungshäuser die wahren medienpädagogischen Verhältnisse in der Erwachsenenbildung vor Ort. Dies bestätigt die Analyse der Veranstaltungsverzeichnisse nachdrücklichst. Vor Ort an der Basis erschöpfen sich medienpädagogische Bemühungen nur zum verschwindend geringen Teil nicht im Einzelabend. Von den 63 Veranstaltungseinheiten, welche die Sichtung der Arbeitspläne für das 1. Halbjahr 1980 registrierte, waren allein 57 - also rund 90 Prozent - Maßnahmen, die nur einen Abend dauerten. Von den 152 Angeboten, die insgesamt in allen drei Erhebungsschritten erfaßt wurden, waren 135 Einzelveranstaltungen.

Wird die Kurzatmigkeit medienpädagogischer Bildungsarbeit durch eine kontinuierliche Programmplanung aufgefangen? Es wäre ja denkbar, daß vor Ort innerhalb eines Arbeitshalbjahrs jeweils nur an einem Abend, dafür aber über mehrere Programmhalbjahre hinweg das Angebot gemacht wird, sich Medienfragen zu stellen, und die Analyse nur eines Veranstaltungsverzeichnisses im Dunkel läßt, ob Medienerziehung in der katholischen Erwachsenenbildung - wenn auch quantitativ relativ bescheiden - letztlich doch systematisch geplant wird.

Aus diesem Grund wurde von 20 Bildungswerken neben dem Veranstaltungsverzeichnis I/80 auch das der Programmhalbjahre I/79 und II/79 berücksichtigt. Die Sichtung erbrachte keinerlei Anhaltspunkte dafür, daß seitens der katholischen $\mathrm{Er}$ wachsenenbildung vor Ort systematisch und kontinuierlich Medienerziehung betrieben wird. Es wurden lediglich fünf von $607 \mathrm{Gemeinden/Pfarreien} \mathrm{gefunden,}$ in denen in mindestens zwei der drei untersuchten Programmhalbjahre medienpädagogische Veranstaltungen angesetzt waren. In zwei aufeinander folgenden Halbjahren hatten nur zwei Gemeinden/Pfarreien vorgesehen, Aspekte der Medien zu thematisieren.

Wie dünn und sporadisch die medienpädagogischen Aktivitäten der katholischen Erwachsenenbildung vor Ort gestreut sind, läßt besonders nachdrücklich die oben angesprochene Längsschnittanalyse des örtlichen Bildungsprogramms von $100 \mathrm{Ge}$ meinden/Pfarreien in den zurückliegenden zehn Programmhalbjahren deutlich werden. Insgesamt wurden dabei $53 \mathrm{Gemeinden/Pfarreien} \mathrm{ausfindig} \mathrm{gemacht,} \mathrm{in}$ deren Arbeitsplänen in den zurückliegenden fünf Jahren keinerlei medienpädagogische Bemühungen auszumachen waren, die sich in eigenen medienpädagogischen Veranstaltungsangeboten niedergeschlagen hätten. Im Programm von 41 Gemeinden aus den letzten fünf Jahren fand sich lediglich ein medienpädagogisches Angebot, in sechs zwei und mehr Angebote, und die noch allesamt nicht aufeinanderfolgend, sondern immer nach medienpädagogischen Zwischenpausen und auch von der Thematik her eher beziehungslos geplant. 


\section{$Z u$ den Zielen und Inhalten}

Was im einzelnen in den Veranstaltungen thematisiert und problematisiert wurde und wird, entzieht sich selbstverständlich der Analyse von Programmheften. Sie kann lediglich aus den Themenformulierungen, etwaigen Untertiteln und etwaigen erläuternden Angaben grobe Rückschlüsse auf Zielakzente und Inhaltsgewichtungen ziehen und über diesen Weg erste Antworten auf die Frage versuchen, unter welchem Blickwinkel die katholische Erwachsenenbildung den Lerngegenstand Massenmedien sieht und präsentiert. Um es gleich vorweg zu nehmen: Auch inhaltlich zeigt das Angebot eine hochgradige Gleichförmigkeit.

Sie äußert sich zum einen in einer starken Konzentration auf das Medium Fernsehen: Von den 63 medienpädagogisch klassifizierten Bildungseinheiten, die sich in den 40 untersuchten Bildungswerken im ersten Programmhalbjahr 1980 fanden, hatten nicht weniger als 41 bereits im Titel einen ausdrücklichen Bezug zum Fernsehen. Von allen in den verschiedenen Erhebungsschritten erfaßten 152 Veranstaltungen waren es 99 , also knapp zwei Drittel. Im 1. Halbjahr 1980 beschäftigten sich dagegen nur neun Veranstaltungen mit der Presse/Zeitung, insgesamt von allen 152 Veranstaltungen nur 24. In derselben Größenordnung bewegt sich das Veranstaltungsangebot, das sich allgemein mit „Massenmedien” befaßte.

Auffallend auch bei einer ersten oberflächlichen Durchsicht der Themenliste die dominierende Perspektive, von der aus katholische Erwachsenenbildung Massenmedien sieht: Nicht weniger als 81 der 152 erfaßten Veranstaltungen stellten bereits im Titel einen ausdrücklichen Bezug zu Kind oder Jugendlichen her. „Mein Kind und das Fernsehen”, „Kinder vor dem Fernsehen”, „Fernsehen - Hilfe oder Heimsuchung für unsere Kinder”, „Was lesen unsere Kinder?” - so oder ähnlich lauteten über die Hälfte aller Themen. Rechnet man noch die 17 Veranstaltungen mit, die Massenmedien in Bezug zur Familie brachten, so tritt der Primat der pädagogischen Perspektive noch deutlicher zutage.

Massenmedien werden in der katholischen Erwachsenenbildung vor Ort vornehmlich als potentieller Stör- oder Förderfaktor kindlicher Entwicklung angesehen, also unter dem Gesichtspunkt möglicher positiver oder negativer Wirkung behandelt, und wie es scheint, weit mehr unter dem negativer Wirkung. Dieser Eindruck wird noch dadurch erhärtet, da $\beta$ im Restangebot an Veranstaltungen in 18 Fällen im Titel bzw. den Veranstaltungserläuterungen auf die Manipulations- und Verdummungsgefahren in der Berichterstattung angespielt wird.

Demgegenüber bleiben Veranstaltungen, die Wesen und Funktion der Medien als „Instrumente sozialer Kommunikation” durchsichtiger zu machen, die Einblick in ihre Struktur und Organisationsform zu vermitteln suchen, sehr dünn gesät. Ein Großteil der Einzelangebote, die eher einer solchen Globalzielkategorie zuzuschlagen wären, rekrutierte sich aus Exkursionen in Rundfunkhäusern, Regionalstudios und Zeitungsverlagen. Insgesamt boten etwa zehn Prozent der erfaßten medienpädagogischen Einheiten die Möglichkeit, über die Entstehung einer Zeitung, einer Fernsehsendung an Ort und Stelle, sozusagen aus erster Hand etwas zu erfahren.

Auch Veranstaltungen zu aktuellen Entwicklungen in der Kommunikationslandschaft scheinen vor Ort nicht stattzufinden. Die in den letzten Jahren stark aufgekommene öffentliche Diskussion um die Einführung neuer Kommunikationsmedien und um etwaige Veränderungen im Fernsehprogramm-Status quo haben 
beispielsweise im ersten Halbjahresprogramm 1980 nur in einer einzigen Veranstaltung vor Ort ihren Niederschlag gefunden. 1979 wurde die Thematik in den untersuchten 20 Bildungswerken überhaupt nicht angesprochen.

Wie gesagt, die Zielvorstellung der in der katholischen Erwachsenenbildung angestellten Versuche zur Medienerziehung läßt sich selbstverständlich aus den Programmverzeichnissen allein nicht im Detail zuverlässig herausfiltern. Aber die dort gemachten Beobachtungen legen den Verdacht nahe, daß katholische Erwachsenenbildung mit einer verkürzten medienpädagogischen Zielvorstellung operiert. Medien werden überwiegend unter dem Blickwinkel ihres Wirkungspotentials gesehen. Medienpädagogik wird damit verstanden als Erziehung zum pädagogisch wertvollen Auswählen aus dem publizistischen Angebot, als Erziehung zum ,rechten" Konsum, zum guten Hörer, Leser und Zuschauer, und weniger als Einübung in die Rolle des aktiven Kommunikationspartners im Gespräch der Gesellschaft.

\section{Zweifel am Lernertrag}

Die Erhebung hat nur Schlaglichter auf die medienpädagogischen Aktivitäten katholischer Erwachsenenbildung werfen wollen und können. Was dabei an Konturen sichtbar wurde, legt allerdings eine tiefe Kluft zwischen Anspruch und Wirklichkeit frei - in quantitativer wie in qualitativer Hinsicht. Medienpädagogische Angebote fristen nicht nur ein kümmerliches Randdasein unterhalb der 1-ProzentMarke im Gesamt der Bildungsangebote vor Ort. Sie wirken auch völlig zufällig und beliebig. Und was sich vereinzelt finden läßt, erscheint in seiner Zielsetzung - gemessen an den in der Pastoralinstruktion Communio et progressio von der Kirche proklamierten Grundsätzen - verkürzt. Man wird deshalb die faktische Leistung katholischer Erwachsenenbildung vor Ort im Dienst der Befähigung zu aktiver Teilhabe am Kommunikationsgeschehen als äußerst geringfügig veranschlagen dürfen.

Ja, angesichts der hochgradigen Kurzatmigkeit, von der die allermeisten vor Ort vorfindbaren medienpädagogischen Versuche befallen sind, drängen sich massive Zweifel auf, ob sie überhaupt nennenswerte Bildungswirkung in Sachen Medien hinterlassen, ungeachtet der Verengungen des Medienbegriffs, mit dem sie operieren. Müssen an einem Abend komplizierte Sachverhalte, Wirkungszusammenhänge, Forschungsbefunde nicht häufig versimplifiziert werden? Drängt das Kurzangebot nicht allzu sehr einfache Ratschläge im Umgang mit dem Fernsehen auf, Rezepte sozusagen, die im Alltag nicht tragen? Sind Konsumgewohnheiten und Verhaltensmuster, die sich in jahrelangen Vorgängen eingeschliffen haben, an einem Abend dauerhaft aufzubrechen? Steht hinter einer solchen Programmgestaltung nicht der Glaube an eine lineare Ursache - Wirkungs-Beziehung im kommunikativen Geschehen - hier die Veranstaltungsbotschaft und dort die entsprechende Verhaltensänderung - ein Glaube, den die Kommunikationsforschung längst als naive Illusion entlarvt hat?

Bei der Einschätzung der Wirkung medienpädagogischer Aktivitäten der katholischen Erwachsenenbildung müssen überdies aus der Kurzatmigkeit resultierende lernerfolgsbeeinträchtigende methodische Zwänge in Rechnung gestellt werden: Die Ein-Abend-Bildung erschwert in aller Regel den Einsatz kommunikativer Lehr-und Lernformen. Referent und Teilnehmer kennen sich zumeist nicht. Das errichtet Hemmschwellen - beim Referenten, teilnehmeraktivierend $\mathrm{zu}$ arbeiten, bei den Teilnehmern, sich mit den eigenen Erfahrungen, Ansichten, Beobachtun- 
gen, Problemlösungsvorschlägen in das Veranstaltungsgeschehen einzubringen. Kommt der gewaltige Stoffberg hinzu, den viele Themen implizieren, der wenig Zeit für zeitraubendes selbstentdeckendes Lernen läßt. Die Folgen sind vielfach große Dominanz des Referenten und Konsumhaltung der „Hörer”, eine ungünstige Lernkonstellation.

Das drängt die Frage auf, wie die Fähigkeit zur Kommunikation, zur Zusammenarbeit und zur Mitgestaltung des sozialen Geschehens vermittelt werden kann, wenn sie schon während der Veranstaltung aus Gründen der Kurzatmigkeit nicht intensiv eingeübt wird. Von einer Position aus, die in der Medienpädagogik das Instrument zur besseren, sachgerechteren Wahrnehmung der Rolle als Partner im gesellschaftlichen Gespräch sieht - und die amtskirchlichen Grundsätze zum Engagement im medienpädagogischen Bereich haben diese Position -, nehmen sich die der Programmplanung innewohnenden methodischen Zwänge zum Referentenmonolog und zur Publikumshaltung besonders widersinnig aus.

Überdies muß in Rechnung gestellt werden, daß in der Bildungsarbeit vor Ort der Lernerfolg weitgehend nur von der Veranstaltungsteilnahme abhängig ist, d.h. aber vom gesprochenen und sich rasch wieder verflüchtigenden Wort. Häusliche Nacharbeit anhand vertiefender weiterführender Materialien ist ihr fremd. Solche Ausgangsbedingungen lassen befürchten, daß häufig nur Inseln punktuellen Faktenund Rezeptwissens in Sachen Medien aufgebaut werden, die infolge ihrer Isoliertheit wieder verschwinden.

Angesichts sich abzeichnender Veränderungen in der Medienlandschaft, welche die Bedeutung der Medien im Leben des Bundesbürgers aller Voraussicht nach noch vergrößern, rufen die Defizite und Schwächen im medienpädagogischen Engagement der katholischen Erwachsenenbildung vor Ort nach verstärkten Bemühungen um Abhilfe. Geht man davon aus, daß sie Ausdruck, Produkt, Symptome tieferliegender Defizite und Schwächen sind, so wird es zunächst einmal entscheidend darauf ankommen, das System katholischer Bildungsarbeit sorgfältig auf Punkte und Mechanismen hin zu durchleuchten, die den seit Jahren angestrebten quantitativen und qualitativen Ausbau der Medienerziehung blockieren. Auch im Interesse der Glaubwürdigkeit des gesellschaftlichen Handelns der Kirche erscheint die Suche nach den Hintergründen für das krasse Auseinanderfallen ihrer medienpädagogischen Selbstansprüche und der wirklichen Leistung im Bereich ihrer Bildungsarbeit mit Erwachsenen dringlich.

\section{Anmerkungen}

1 Franz Henrich: Erwachsenenbildung in katholischer Trägerschaft. In: „Stimmen der Zeit", Heft 6/1972, S. 405 ff.

2 Kirchlicher Erwachsenenbildungsplan für die Erzdiözese München und Freising. München 1976, S. 5 f.

3 Plan für die offene Erwachsenenbildung in der Diözese Eichstätt. Eichstätt 1980, S. 7.

4 Georg Moser: Zur pastoralen Bedeutung der Erwachsenenbildung. In: Kath. Landesarbeitsgemeinschaft für Erwachsenenbildung Baden-Württemberg (Hrsg.): Jahrbuch 1973, S. 27. 
5 Stellungnahme des Zentralkomitees der Deutschen Katholiken zu aktuellen Fragen der Weiterbildung, vom 22. Juni 1979, hektografierte Fassung, S. 2.

6 Pastoralinstruktion „Communio et Progressio" über die lnstrumente der sozialen Kommunikation (in der Fassung: Nachkonziliare Dokumentation, Band 11, Trier 1971), Nr. 107 (S. 237).

7 Ebenda, Nr. 66 (S. 207).

8 Ebenda, Nr. 65 (S. 207).

9 Vgl. Hans Wagner: Einführung und Kommentar. In: Pastoralinstruktion ..., a.a.O., S. 101.

10 Franz Henrich: Überlegungen zu einem Konzept katholischer Erwachsenenbildung, hrsg. von der Katholischen Bundesarbeitsgemeinschaft für Erwachsenenbildung. Bonn 1979 S. 18.

II Vgl. Katholische Bundesarbeitsgemeinschaft für Erwachsenenbildung (Hrsg.): Statistische Berichte 1979. Bonn 1979, S. 22 ff.

12 Vgl. Statistische Berichte des Bayerischen Statistischen Landesamts, Erwachsenenbildung in Bayern 1977. München 1978, S. 15 ff.

$13 \mathrm{Vgl}$. Katholische Bundesarbeitsgemeinschaft für Erwachsenenbildung (Hrsg.): Statistische Berichte 1979, S. 49 ff.

14 Vgl. Bildungswerk der Diözese Limburg (Hrsg.): 20 Jahre Bildungswerk der Diözese Limburg, Limburg o.J., S. 86.

$15 \mathrm{Vgl}$. Bildungswerk der Erzdiözese Freiburg (Hrsg.): Erwachsenenbildung als kirchliche Aufgabe. Freiburg 1980, S. 41.

16 Die hier und im folgenden für Bayern und Hessen angegebenen Zahlen entstammen der in Anmerkung 11 genannten Quelle.

\section{SUMMARY}

Media education is a need which can no longer be ove rlooked. University Chairs for teaching such subjects have been established. In schools, the use of media is part of the normal curriculum. How is this subject being dealt with in Adult Education programmes in Germany? According to statistics, the conclusion must be drawn that there is a wide gap between supply and demand. Options for media education in Adult Education programmes are still only marginal, occasional and random, and, in the light of Communio et Progressio, these fall short of what is required. They are often limited to single areas and concerns. This lacunae in Catholic education has to be remedied as soon as possible. 


\section{RÉSUMÉ}

De nos jours, la pédagogie des mass media a une côte d'une grande urgence qu'on ne peut pas ne pas remarquer. Dans l'instruction publique, les mass media font depuis longtemps partie de l'enseignement. Qu'en est-il de cela dans l'éducation pour adultes? L'auteur examine cette question à l'aide de recherches statistiques. Si leur quadrillage est à l'avenir également grossier et n'éclaircit la situation que de façon subite, alors ici apparaît un gouffre profond entre l'exigence et la réalité. Les offres pédagogiques des mass media mettent un terme à une existence en marge, agissent par hasard, quelconques - et mesurés aux principes de Communio et Progressio - raccourcis. Par le mot parlé résultent souvent seulement des îles de faits et de savoir-formules ponctuels. Les déficits et les faiblesses dans l'enseignement pédagogique des mass media de formation catholique pour adultes locale appellent à un effort renforcé pour chercher des remèdes.

\section{RESUMEN}

La pedagogía de los medios de comunicación social es hoy un desideratum no despreciable y de urgencia. Para ella fue ron creadas cátedras. En las escuelas los medios de comunicación forman parte, desde hace tiempo, de los planes de enseñanza. Pero cabe preguntarse que lugar ocupan en la formación de adultos. Este es el tema que analiza el autor a base de estadísticas. Su retículo es todavía incompleto y por ello se produce un profundo desfase entre aspiración y realidad. Las ofertas pedagógicas sobre medios de comunicación social prorrogan marginalmente, operan casualmente, recortadas a discreción y medidas a tenor de los criterios de la Communio et Progressio. Mediante la palabra hablada solo surgen a menudo islas de conocimientos aislados. Los déficits y lagunas en el compromiso pedagógico para la fórmación de adultos católicos en materia de comunicación social piden mayor esfuerzo para encontrar remedios. 